\title{
The Application of Urine Tests to Monitor the Regularity of Dapsone Self-administration
}

\author{
G. A. ELLARD AND PATRICIA T. GAMMON \\ Medical Research Council's Unit for Laboratory Studies of Tuberculosis, \\ Royal Postgraduate Medical School, London W12 OHS, England \\ and \\ JUNE M. HARRIS \\ Somerville College, Oxford $O X 26 H D$, England
}

\begin{abstract}
Simple methods are described for determining the regularity of dapsone (DDS) self-administration by out-patients. These methods are based on a comparison of the ratios of the concentrations of DDS to those of creatinine in urine samples collected from out-patients and those from controls given the same daily dose of DDS under strict supervision. These methods were applied to urine samples collected from patients attending some of the mobile clinics in Malawi. They showed that the patients had taken only about half of their prescribed DDS doses in the days immediately preceding their attendance at the clinic. The therapeutic importance of these findings is discussed, and the value of extending these studies to obtain estimates of the regularity of DDS self-administration through the whole treatment period by applying such methods to urine samples obtained by surprise visits to the homes of the out-patients is emphasized.

The mean rate of elimination of DDS determined among a small group of Malawian,patients was equivalent to a half-life of about $31 \mathrm{~h}$. Significant differences were found between patients in the rates at which they eliminated DDS but these differences were unrelated to the extent to which they acetylated the drug.
\end{abstract}

\section{Introduction}

Dapsone (DDS) remains the drug of choice for the world-wide treatment of leprosy (Ellard, 1974). Since most patients receive their treatment from out-patient clinics, the successful mass treatment of leprosy is therefore very largely dependent on the regularity with which out-patients take the DDS tablets they are given. Experience in the treatment of other diseases indicates that self-medication is often unreliable (Fox, 1962; Porter, 1968). Thus recent studies of the treatment of pulmonary tuberculosis in East Africa, Hong Kong and India have all demonstrated that drug regimens that are highly successful in controlled clinical trials often fail when used routinely in large-scale treatment because 
patients fail to take their drugs (Fox, 1968, 1972; Kent et al., 1970; Hong Kong Treatment Services/British Medical Research Council, 1972). Similar problems inevitably occur in the mass treatment of leprosy. For instance Pettit and Rees (1964) showed that 4 out of 7 patients who apparently failed to respond to sulphone treatment of over 13 years duration, still harboured DDS-sensitive Mycobacterium leprae. During much of this period these patients presumably had often failed to take their prescribed treatment, and when their dapsone treatment was fully supervised a satisfactory therapeutic response was obtained.

In a number of recent controlled clinical trials, simple qualitative urine tests have been used to assess the regularity with which tuberculosis patients took their prescribed medicaments (British Medical Research Council Co-operative Study, 1973; Hong Kong Treatment Services/British Medical Research Council, 1972; Nazareth et al., 1971; Singapore Tuberculosis Service/Brompton Hospital/British Medical Research Council Investigation, 1971; Tuberculosis Chemotherapy Centre, Madras, 1970, 1973; WHO Collaborating Centre for Tuberculosis Chemotherapy, Prague, 1971). In several of the studies patients with higher proportions of negative urine tests were shown to have had a significantly poorer response to chemotherapy.

We have recently evaluated the potential use of several urine-test methods for monitoring the regularity of DDS self-administration (Ellard et al., 1974a). Methods based on the detection of diazotizable compounds in the urine or on their quantitative determination were found to be unsatisfactory because of the marked effects of diuresis on the urinary concentrations of DDS, its diazotizable metabolites and of natural diazotizable compounds. The effects of diuresis could however be largely overcome by determining the ratios of the urinary concentrations of diazotizable compounds (as DDS) to those of creatinine, a normal urinary constituent whose rate of excretion in subjects varies little from day to day or from hour to hour. By comparing the ratios of the concentrations of DDS/creatinine in urine samples from patients self-administering their DDS with those from patients receiving the same daily dose of DDS under strict supervision, and from controls not taking DDS, the proportion of self-administered DDS doses actually being taken can be calculated (Ellard et al., 1974a). For this purpose urine samples should if possible be obtained by means of surprise visits to the homes of out-patients self-administering their DDS. Because of the slow elimination of DDS from the body and the large range of DDS/creatinine ratios found in urine samples from different patients receiving the same daily dose of DDS, the result of a single urine test cannot establish conclusively whether or not a particular patient actually took their last prescribed dose. This question can however be answered by making a comparison with the results obtained when the patient is given the same dose under supervision.

This paper describes the results obtained when the DDS/creatinine ratios of urine samples collected from out-patients in Malawi who were self-administering their daily DDS doses, and from in-patients receiving the same DDS dosage under supervision, were determined, using simple colorimetric methods. It also describes an alternative procedure for determining the proportion of DDS doses actually being taken by the out-patients, in which pools were prepared from urine samples from the two groups of patients and the DDS/creatinine ratios of the pools then determined using a specific fluorimetric method for DDS. The rates of elimination of DDS from the plasma by a small group of Malawian patients were also determined. 


\section{Methods}

\section{Collection of samples}

Control urine samples were collected from each of 12 patients in the leprosy wards of the LEPRA Control Centre, Blantyre, Malawi, $24 \mathrm{~h}$ after they had taken at least four consecutive supervised daily doses of $25 \mathrm{mg}$ DDS, and from 12 healthy staff who were not taking DDS. A urine sample was also collected from each of 206 male patients attending the fortnightly out-patient clinics served by the Land Rover circuits administered by the LEPRA Control project, Blantyre ("mobile clinic" samples). At each visit to these clinics patients are given a fortnight's supply of $25 \mathrm{mg}$ DDS tablets for daily self-administration. Each patient was questioned as to when his last DDS dose had been taken. Thirty-seven patients stated they had taken their last DDS dose earlier in the day, and since possible absorption of DDS from this dose would have invalidated the comparison with the control urines, these samples were subsequently discarded, leaving a total of 169 for analysis. Nine other patients failed to produce a urine sample. Similar urine samples were also obtained from 15 patients attending the Blantyre out-patient clinic. Each urine sample was preserved by the addition of approximately 0.5 volumes of $2 \mathrm{~N}-\mathrm{HCl}$ and stored without refrigeration until analysis in London three months later.

Four blood samples were collected from each of 17 leprosy patients in the LEPRA Control Centre, Blantyre, at 4 and $24 \mathrm{~h}$ respectively after two consecutive daily doses of $50 \mathrm{mg}$ DDS had been taken on an empty stomach. The blood was taken into heparinized tubes, the plasma rapidly separated off and then stored at $-20^{\circ} \mathrm{C}$ until analysis in London.

\section{Analyses}

Urinary concentrations of total diazotizable compounds (as DDS) and of creatinine were determined by the modifications of the Bratton and Marshall (1939) and alkaline picrate methods described previously (Ellard et al., 1974a). Plasma concentrations of DDS and monoacetyl-DDS (MADDS) were determined fluorimetrically by a simplified procedure based on our previous method (Ellard and Gammon, 1969). In this modification $3 \mathrm{ml}$ of serum was extracted with $15 \mathrm{ml}$ ethyl acetate after the addition of $1 \mathrm{ml} \mathrm{M}$-tri-sodium citrate. Ten $\mathrm{ml}$ of the ethyl acetate extract was then washed successively by shaking in the same stoppered centrifuge-tube with $1 \mathrm{ml}$ aliquots of $0.1 \mathrm{~N}$-sodium hydroxide, $0.1 \mathrm{~N}-\mathrm{HCl}$ and $\mathrm{m}$-sodium citrate, respectively. After drying the washed ethyl acetate extract with $1 \mathrm{~g}$ anhydrous sodium sulphate the concentration of DDS was determined from the fluorescence at $298 / 345 \mathrm{~nm}$, and that of MADDS from the fluorescence at $298 / 420 \mathrm{~nm}$, after allowing for the contribution due to DDS. The fluorescence of the ethyl acetate extracts were also determined at 295/324 nm.

\section{Analyses of special samples}

The possibility that some of the patients might have been taking sulphonamides concomitantly was suggested by the fact that several of the urine samples had considerably higher DDS/creatinine ratios than those encountered in urine samples from Malaysian patients receiving $25 \mathrm{mg}$ DDS daily under strict supervision (Ellard et al., 1974a). To clarify the situations further analyses were undertaken on all the urine samples that were originally estimated to contain 
more than $40 \mu \mathrm{g}$ DDS/mg creatinine. The colorimetric creatinine and DDS determinations were first repeated and the reaction product obtained in the DDS method scanned in a recording spectrophotometer. Aliquots of the acidified urine samples were also neutralized and extracted by the procedure used for the fluorimetric determination of DDS (Ellard and Gammon, 1969) and the extracted DDS determined both fluorimetrically and colorimetrically (Ellard et al., 1971).

\section{Analyses of representative pools of urine samples}

Pools were constituted either by mixing equal volumes of urine, or by mixing volumes of urine containing equal amounts of creatinine as determined colorimetrically by the alkaline picrate method. Pools were prepared in this way from urine samples obtained from the healthy staff not taking DDS, from the control patients receiving supervised daily doses of $25 \mathrm{mg}$ DDS and from the out-patients served by the mobile clinics. One ml aliquots of these pools were made alkaline by the addition of $1 \mathrm{ml} \mathrm{N}$-sodium hydroxide and DDS determined fluorimetrically (Ellard and Gammon, 1969). Their creatinine concentrations were also determined.

\section{Results}

\section{Analyses of special samples}

The repeat colorimetric determinations carried out on the 13 urine samples found to have ratios of greater than $40 \mu \mathrm{g} \mathrm{DDS} / \mathrm{mg}$ creatinine confirmed the original results in every case. In five of the samples, with apparent DDS/creatinine ratios of 61-135 (mean 99), there was evidence for the presence of significant concentrations of diazotizable compounds other than DDS and its metabolites, presumably because the out-patients concerned had been taking sulphonamides. Thus when these five samples were reacted directly by the modified Bratton and Marshall procedure, the absorption of the reaction product at $550 \mathrm{~nm}$ was more than twice that of $600 \mathrm{~nm}$ in direct contrast to the results from the other eight samples with DDS/creatinine ratios of over 40 (range 41-79, mean 53) and the 11 samples from the supervised controls with ratios of less than 40 (range 18-36, mean 27). Furthermore the concentrations of fluorimetrically-determined DDS in these five samples averaged only about $10 \%$ (range 3-15\%) of the concentrations of diazotizable compounds determined by the direct colorimetric method, compared to an average of about 50\% (range 30-70\%) for the other samples. In two of the five samples the interfering sulphonamide did not appreciably extract in the solvent system used for the fluorimetric determination of DDS and the extracts obtained reacted and fluoresced in an identical fashion to DDS. The other three samples contained sulphonamides that were partially extracted into ethyl acetate and thence into $1.2 \mathrm{~N}-\mathrm{HCl}$. On diazotization and coupling they gave a sharp peak at $550 \mathrm{~nm}$ unlike the flatter peak given by DDS at $570 \mathrm{~nm}$, and they were less fluorescent in ethyl acetate than DDS, their maximal fluorescence occurring at $275 / 340 \mathrm{~nm}$ instead of at $298 / 345 \mathrm{~nm}$ as is found for DDS.

\section{Colorimetrically-determined DDS/creatinine ratios}

The results obtained, after excluding the values for the five samples for which convincing evidence of interfering sulphonamides was obtained, are summarized in Table 1. The DDS/creatinine ratios of the 164 urine samples from the 
TABLE 1

Ratios of DDS/creatinine determined colorimetrically in urine samples from out-patients and controls

\begin{tabular}{|c|c|c|c|c|}
\hline \multirow[t]{2}{*}{ Origin of samples ${ }^{a}$} & \multirow{2}{*}{$\begin{array}{l}\text { Number of } \\
\text { subjects }\end{array}$} & \multicolumn{2}{|c|}{ DDS/creatinine ratios } & \multirow{2}{*}{$\begin{array}{l}\text { Estimated } \% \text { doses } \\
\text { taken }^{c}\end{array}$} \\
\hline & & Range & Mean $^{b}$ & \\
\hline Controls not on DDS & 12 & $2.6-10.9$ & $5.2 \pm 0.7$ & 0 \\
\hline Supervised controls on DDS & 12 & $17.6-45.5$ & $28.6 \pm 2.3$ & 100 \\
\hline \multicolumn{5}{|l|}{ Out-patients } \\
\hline No default admitted & 101 & $3.7-78.6$ & $21.1 \pm 1.3$ & 68 \\
\hline Default admitted & 63 & $3.1-40.2$ & $11.8 \pm 1.1$ & 28 \\
\hline All out-patients & 164 & $3.1-78.6$ & $17.6 \pm 1.0$ & 53 \\
\hline Taking DDS regularly ${ }^{a}$ & 82 & $15.1-78.6$ & $26.4 \pm 1.2$ & 91 \\
\hline Taking DDS irregularly $^{a}$ & 33 & $10.0-14.9$ & $12.3 \pm 0.3$ & 30 \\
\hline Taking DDS grossly irregularly ${ }^{a}$ & 49 & $3.1-9.6$ & $6.1 \pm 0.2$ & 4 \\
\hline
\end{tabular}

${ }^{a}$ For details and definitions see text.

${ }^{b}$ Mean \pm standard error of mean $(\mu \mathrm{g} / \mathrm{mg})$.

$c \frac{\text { Mean test ratio-mean blank ratio }}{\text { Mean control ratio- mean blank ratio }} \times 100$.

out-patients attending the mobile clinics averaged $17.6 \pm 1.0$ compared to $28.6 \pm 2.3$ for the controls receiving the same daily dose of DDS under strict supervision and $5.2 \pm 0.7$ for the healthy subjects not taking DDS. It was therefore calculated that in the immediate period before the urine samples were collected only about 53\% (17.6-5.2/28.6-5.2 × 100) of their prescribed DDS doses had been taken by the out-patients. A similar proportion (57\%) was calculated from the ratios of the 15 samples from out-patients attending the Blantyre clinic. When the urine samples collected at the mobile clinics from patients who claimed to have taken their last DDS dose on the previous day were considered, the estimated proportion of doses taken rose to $68 \%$, in contrast to a value of only $28 \%$ for those who admitted failing to take a dose of DDS on the previous day. There was however no significant difference between the results from the 11 patients who admitted to only a single missed dose and those from the 52 who confessed to more serious default.

By analogy with the results from the controls, it was considered probable that the 82 out-patients ( $50 \%$ of the total) with DDS/creatinine ratios of greater than 15 had taken their prescribed DDS doses regularly in the days immediately prior to the collection of the urine samples, that the $33(20 \%)$ with ratios of $10-15$ might have been irregular, and that the $49(30 \%)$ with ratios of less than 10 might have been grossly irregular in their drug taking. The proportions of prescribed DDS being taken by these three groups calculated from their mean DDS/ creatinine ratios were about $91 \%, 30 \%$ and $4 \%$, respectively (Table 1 ).

\section{Fluorimetric analysis of representative pooled urines}

The pools prepared from the urine samples collected from the 12 healthy staff not taking DDS yielded ethyl acetate extracts with negligible fluorescence at the wavelengths used to determine DDS, while the pools prepared from the controls 
given supervised DDS doses and the out-patients served by the Land Rover circuits yielded extracts whose fluorescence characteristics were identical to those of DDS. The ratios of fluorimetrically determined DDS/creatinine in these pools are summarized in Table 2. Almost identical results were obtained whether the pools were prepared by mixing equal volumes of urine or by mixing aliquots containing equal amounts of creatinine. Furthermore it was found that the results were unaffected by the inclusion of the five samples containing sulphonamides in the pools. From these results it was calculated that the out-patients had been taking only $52-53 \%$ of their prescribed DDS doses during the period immediately before the urine samples were collected. Analyses of the urine pools prepared from samples with DDS/creatinine ratios of less than 10, between 10 and 15, and over 15 , respectively, revealed that in this period about $6 \%$ of the prescribed DDS doses had been taken by the "grossly irregular" patients, 33-36\% by the "irregular" patients and $91 \%$ by the "regular" patients. These findings were in remarkable agreement with those calculated from the means of the individual colorimetrically-determined ratios for the three groups.

\section{Plasma concentrations of DDS and MADDS}

There were highly significant differences between patients in the ratios of the plasma concentrations of MADDS/DDS attained at 4 and $24 \mathrm{~h}$ after dosage with $50 \mathrm{mg}$ DDS, eight of the patients being slow acetylators with mean MADDS/DDS ratios of 0.16 (range 0.12-0.18) and nine rapid acetylators with mean MADDS/DDS ratios of 0.46 (range 0.34-0.55). In any one patient however the ratios attained at 4 or $24 \mathrm{~h}$ were very similar. The mean half-lives of DDS of the 17 patients calculated from the fall in DDS plasma concentrations from 4-24 h after giving two successive doses of DDS averaged $31 \mathrm{~h}$ (range 25-43 h). Significant differences were found between patients in the rates of elimination of DDS $(P=0.025)$, but these differences were unrelated to the extent to which the patients acetylated DDS. The fluorescence of the ethyl acetate extracts of the plasma samples at 295/324 nm equalled that due to the calculated concentrations of DDS and MADDS, indicating that significant concentrations of diacetyl-DDS (DADDS) were not present.

\section{Discussion}

The remarkable agreement between the estimated proportions of the prescribed DDS doses being taken by the out-patients served by the mobile clinics, whether calculated from the means of the individual colorimetrically-determined DDS/creatinine ratios or from the ratios of fluorimetrically-determined DDS/creatinine of the representative pools of the urine samples (Table 2), indicates the validity of either method for monitoring the regularity of DDS self-administration. To prepare truly representative urine pools, volumes of urine containing equal amounts of creatinine should be mixed. However, since no correlation was found between the colorimetrically-determined DDS/creatinine ratios of the urine samples and their creatinine concentrations, it is understandable that virtually identical results were obtained whether the pools were prepared by mixing aliquots of equal creatinine content or much more conveniently by mixing aliquots of equal volume. It is of interest that the colorimetrically-determined DDS/creatinine ratios of the Malawian controls not taking DDS and of those taking supervised daily doses of $25 \mathrm{mg}$ DDS were 
TABLE 2

Comparison of the results of three different methods of assessing the percentage of DDS doses actually being taken

\begin{tabular}{|c|c|c|c|c|c|c|}
\hline \multirow{3}{*}{ Sources of samples ${ }^{a}$} & \multicolumn{6}{|c|}{ Method } \\
\hline & \multicolumn{2}{|c|}{$\begin{array}{l}\text { Individual estimations } \\
\text { (DDS colorimetric) }^{c}\end{array}$} & \multicolumn{2}{|c|}{$\begin{array}{l}\text { Equal volume pool } \\
\text { (DDS fluorimetric) }^{c}\end{array}$} & \multicolumn{2}{|c|}{$\begin{array}{l}\text { Eq ual creatinine pool } \\
\text { (DDS fluorimetric) }^{c}\end{array}$} \\
\hline & DDS/creatinine & $\%$ doses taken & DDS/creatinine & $\%$ doses taken & DDS/creatinine & $\%$ doses taken \\
\hline Controls not on DDS & $5.2^{b} \pm 0.7$ & 0 & $<0.1$ & 0 & $<0.1$ & 0 \\
\hline Supervised controls on DDS & $28.6 \pm 2.3$ & 100 & 13.1 & 100 & 13.0 & 100 \\
\hline \multicolumn{7}{|l|}{ Out-patients } \\
\hline All out-patients & $17.6 \pm 1.0$ & 53 & 6.8 & 52 & 6.9 & 53 \\
\hline Taking DDS regularly $^{a}$ & $26.4 \pm 1.2$ & 91 & 11.9 & 91 & 11.8 & 91 \\
\hline Taking DDS irregularly $^{a}$ & $12.3 \pm 0.3$ & 30 & 4.7 & 36 & 4.3 & 33 \\
\hline Taking DDS grossly irregularly $^{a}$ & $6.1 \pm 0.2$ & 4 & 0.8 & 6 & 0.8 & 6 \\
\hline
\end{tabular}

${ }^{a}$ For details and definitions see text.

${ }^{b}$ Mean \pm standard error of mean $(\mu \mathrm{g} / \mathrm{mg})$.

$c$ (Mean) test ratio-(Mean) blank ratio

(Mean) control ratio-(Mean) blank ratio $\times 100$. 
approximately double the corresponding values obtained from Malaysian patients (Ellard et al., 1974a). This finding emphasizes the importance of comparing the results from out-patients with appropriately matched controls.

The apparent ingestion of sulphonamides by some $3 \%$ of the out-patients slightly complicated this investigation. Fortunately the results obtained indicated that such interference could simply be established by measuring the absorption of the reaction product in the colorimetric DDS method at $600 \mathrm{~nm}$ as well as at $500 \mathrm{~nm}$.

The calculated proportion of prescribed DDS doses taken by the out-patients will be a true estimate if the timing of the collection of the samples from the out-patients was the same as that in the controls and if the proportion of DDS doses taken by the out-patients in the days immediately previous to the collection of urine samples was typical. The studies carried out on Malaysian patients showed that after giving a single dose of DDS, DDS/creatinine ratios fell in the urine at similar rates to plasma DDS concentrations (Ellard et al., 1974a; Gelber and Rees, 1974). It may therefore be concluded that differences in timing between the collection of the urine samples from the out-patients served by the mobile clinics (possibly 12-30 h after the previous day's dose) and the control in-patients ( $24 \mathrm{~h}$ after the previous dose) could have overestimated the proportion of DDS doses being taken by the out-patients by $5-10 \%$.

A more serious reservation to interpreting the results obtained in this study rests on the fact that representative urine samples were not obtained by means of surprise visits to the out-patients' homes. It might be argued that the stimulus of preparing to attend the clinic might have reminded many of the patients to have taken their DDS in the immediately proceeding days. Conversely it could however be reasoned that if patients either lost of disposed of their tablets, or missed a clinic attendance, the resultant deficit to their stock of DDS tablets would be most likely to result in a failure of self-administration in the days immediately before the next clinic. It would however seem highly probable that the regularity of DDS self-administration by the out-patients who provided the urine samples analysed in this study was superior to that of the other out-patients who failed to attend at the mobile clinics.

Although attempting to categorize the patients according to their regularity of DDS self-administration is necessarily arbitrary, the results did indicate that only about half of the out-patients attending the mobile clinics had taken their DDS tablets regularly in the 2-3 days immediately preceding the clinic. A further $20 \%$ of the patients had probably taken only about a third of their prescribed doses during this time, while the remaining 30\% had taken virtually no DDS at all during this period. If the irregularity of DDS self-administration by the patients studied in this investigation, revealed in the period immediately before the urine samples were collected, was typical of the whole treatment period, it would be likely to seriously jeopardize hopes of curing the great majority of the patients and of eliminating leprosy from the area within the foreseeable future.

The MADDS/DDS ratios of the slow and rapid acetylators among the 17 Malawian leprosy patients studied were very similar to those found among a small group of dermatitis herpetiformis patients being treated with DDS in Britain (Ellard et al., 1974b) and confirm the previous studies of Gelber et al. (1971) and Peters et al. (1972) which showed that DDS, like isoniazid, sulphadimidine and certain other hydrazides and sulphonamides, is polymorphically acetylated in man. The demonstration of significant differences in the rates of elimination of 
DDS by different subjects confirms the investigation of Peters et al. (1972) while it is of interest that the mean half-life of DDS in the 17 Malawian patients investigated in our study $(31 \mathrm{~h})$ is very similar to that of $28 \mathrm{~h}$ found among 21 African patients in Zaire by Peters et al. (1974). The finding that the half-life of DDS is unrelated to the extent of DDS acetylation also confirms evidence obtained in other investigations (Peters et al., 1972; Gelber and Rees, 1974). The absence of significant concentrations of DADDS in the plasma of DDS-treated patients is also in accord with the studies of Murray et al. (1971) who used an extremely specific and sensitive chromatographic-fluorimeter procedure to determine DDS and its acetylated derivatives.

The results obtained in the present study suggest the following procedure for monitoring the self-administration of DDS by leprosy out-patients. Urine samples should be collected from approximately 20 healthy subjects not taking DDS, from 50-100 out-patients, and from about 20 in-patients receiving the same daily dose of DDS under strict supervision. If possible urine samples should be collected from the out-patients by means of surprise visits early in the morning to their homes. The patients should be questioned as to when they had taken their previous DDS dose. Their stock of remaining DDS tablets should also be inspected since a significant excess over the correct number would indicate failure of DDS self-medicated in the previous period, while a shortage would normally result in supplies being exhausted before the next clinic. Surprise home visits would obviously be much more difficult to organize in rural areas such as those served by the mobile clinics in Malawi than in the semi-urban environments of Madras and Hong Kong where many of the controlled antituberculosis trials described earlier were held. Urine samples would be collected from the in-patients $2-4 \mathrm{~h}$ and $24 \mathrm{~h}$ after their fourth supervised daily dose of DDS and strict precautions taken to ensure that during this period they ingested no other drugs. Pools would then be prepared from $1 \mathrm{ml}$ aliquots of the urine specimens from the control groups from the out-patients who stated they had taken their last DDS dose on the previous day, and from those who stated they had taken their last dose at least $2 \mathrm{~h}$ earlier in the day. Samples from patients having taken a dose of DDS within $2 \mathrm{~h}$ would be unsuitable for analysis on account of uncertainty concerning the proportion of the DDS dose absorbed during this period. Aliquots of the pools would then be preserved without refrigeration by the addition of $0.5 \mathrm{vol}$. $2 \mathrm{~N}-\mathrm{HCl}$, and their DDS/creatinine ratios determined using a sensitive and specific fluorimetric method for the determination of DDS. Interference from other fluorescent compounds is very unlikely and in any case their presence would almost certainly be revealed by examining the fluorescence excitation and emission spectra of the extracts.

Such a study should reveal the overall regularity with which DDS was being taken by the out-patients. If it were considered that the irregularity of DDS self-administration was likely to have unacceptable therapeutic consequences, the DDS/creatinine ratios of the individual urine samples should then be analysed by the simple colorimetric methods described. A comparison of the results from the out-patients with those from the controls, taken together with evidence of the possession of grossly incorrect stocks of DDS tablets, would indicate which patients were likely to be the most serious defaulters in self-medication. Specific efforts could then be made to encourage such patients to take their DDS tablets more regularly, further home visits made and urine samples analysed to establish or indicate whether or not the exhortations had been successful. If consistently 
low ratios of DDS/creatinine were still found in samples from individual patients, the regularity of their drug-taking could be established by comparing the results with those obtained after they had been given fully supervised doses of DDS. In many situations such studies would not be feasible for individual patients, and the only practical response to a serious overall level of defaulting might be to consider replacing unsupervised daily chemotherapy by some form of fully supervised intermittent chemotherapy.

It might be argued from this Malawian study that questioning of the patients was in itself an effective method of discovering irregular DDS self-medication since significiantly lower DDS/creatinine ratios were found among those patients who admitted missing doses. Nevertheless the DDS/creatinine ratios of urine samples from 13 of the 101 out-patients who denied missing DDS doses were not significantly different from those of the controls who had taken no DDS. Obviously strenuous effort should first be made among those patients admitting default to encourage them to take their DDS doses more regularly. However such efforts might well result in these patients insisting on a future occasion that they were taking their DDS regularly, even if this were not the case. Hence the importance of basing action in individual cases on evidence provided by determinations of urinary DDS/creatinine ratios.

\section{Acknowledgements}

We should like to thank Drs B. D. Moleworth and R. J. W. Rees for their help in planning and executing this investigation, Drs F. Gjalt Boerrigter and F. Rampen for their assistance in obtaining the blood samples, and Prof. D. A. Mitchison and Dr Wallace Fox for their advice during the preparation of this report. We should also like to express our gratitutde to the anonymous donor whose generous gift to LEPRA made this investigation possible.

\section{References}

Bratton, A. C. and Marshall, E. K., Jr. (1939). A new coupling component for sulfanilamide determination. J. Biol. Chem. 128, 537.

British Medical Research Council Co-operative Study. (1973). Co-operative controlled trial of a standard regimen of streptomycin, PAS and isoniazid and three alternative regimens of chemotherapy in Britain. Tubercle, Lond. 54, 99.

Ellard, G. A. (1974). Recent advances in the chemotherapy of leprosy. Lepr. Rev. 45, 31.

Ellard, G. A. and Gammon, P. T. (1969). A fluorimetric method for the simultaneous determination of $4,4^{\prime}$-diaminodiphenyl sulfone (DDS), $N$-acetyl-DDS (MADDS) and $N, N^{\prime}$-diacetyl-DDS (DADDS) in serum or urine. Int. J. Lepr. 37, 398.

Ellard, G. A., Gammon, P. T., Rees, R. J. W. and Waters, M. F. R. (1971). Studies on the determination of the minimal inhibitory concentration of $4,4^{\prime}$-diamino-diphenyl-sulphone (Dapsone, DDS) against Mycobacterium leprae. Lepr. Rev. 42, 101.

Ellard, G. A., Gammon, P. T., Helmy, H. S. and Rees, R. J.W.(1974a). Urine tests to monitor the self-administration of dapsone by leprosy patients. Amer. J. trop. Med. Hyg. 23, 464.

Ellard, G. A., Gammon, P. T., Savin, J. A. and Tan, R. S. H. (1974b). Dapsone acetylation in dermatitis herpetiformis. Brit. J. Dermatol. 90, 441 .

Fox, W. (1962). Self-medication of medicaments. A review of published work and a study of the problems. Bull. Int. Un. Tuberc. 32, 307.

Fox, W. (1968). Organisational and administrative considerations in the diagnosis and treatment of pulmonary tuberculosis in the developing countries. Tubercle, Lond. 49, 332.

Fox, W. (1972). General considerations in the choice and management of regimens of chemotherapy for pulmonary tuberculosis. Bull. Int. Un. Tuberc. 47, 49.

Gelber, R., Peters, J. H., Gordon, G. R., Glazko, A. J. and Levy, L. (1971). The polymorphic acetylation of dapsone in man. Clin. Pharmacol. Ther. 12, 225. 
Gelber, R. H. and Rees, R. J. W. (1974). Dapsone metabolism in patients with dapsone-resistant leprosy. In preparation.

Hong Kong Tuberculosis Treatment Services/British Medical Research Council Investigation (1972). A study in Hong Kong to evaluate the role of pretreatment susceptibility tests in the selection of regimens of chemotherapy for pulmonary tuberculosis. Amer. Rev. Resp. Dis. 106, 1.

Kent, P. W., Fox, W., Miller, A. B., Nunn, A. J., Tall, R. and Mitchison, D. A. (1970). The therapy of pulmonary tuberculosis in Kenya: A comparison of the results, achieved in controlled clinical trials with those achieved by routine treatment services. Tubercle, Lond. 51, 24.

Murray, J. F., Jr., Gordon, G. R. and Peters, J. H. (1971). A chromatographic-fluorometric procedure for the determination of nanogram quantities of antileprotic sulphones. J. Lab. Clin. Med. 78, 464.

Nazareth, O., Devadatta, S., Fox, W., Menon, N. K., Radhakrishna, S., Rajappa, D., Ramakrishnan, C. V., Somasundaram, P. R., Stott, H., Subbammal, S. and Velu, S. (1971). Two controlled studies of the efficacy of isoniazid alone in preventing relapse in patients with bacteriologically quiescent pulmonary tuberculosis at the end of one year of chemotherapy. Bull. Wld Hlth Org. 45, 603.

Peters, J. H., Gordon, G. R., Ghoul, D. C., Tolentino, J. G., Walsh, G. P. and Levy, L. (1972). The disposition of the antileprotic drug dapsone (DDS) in Philippine subjects. Amer. $J$. trop. Med. Hyg. 21, 450.

Peters, J. H., Gordon, G. R., Levy, L., Storkan, M. A., Jacobson, R. R., Enna, C. D. and Kirchheimer, W. F. (1974). Metabolic disposition of dapsone in patients with dapsone-resistant leprosy. Amer. J. trop. Med. Hyg. 23, 222.

Pettit, J. H. S. and Rees, R. J. W. (1964). Sulphone resistance in leprosy. An experimental and clinical study. Lancet 2, 673 .

Porter, A. M. W. (1968). The problem of the self-administration of drugs. M.D. Thesis University of London.

Singapore Tuberculosis Services/Brompton Hospital/British Medical Research Council Investigation. (1971). A controlled clinical trial of the role of thiacetazone-containing regimens in the treatment of pulmonary tuberculosis in Singapore. Tubercle, Lond. 52, 88.

Tuberculosis Chemotherapy Centre, Madras. (1970). A controlled comparison of a twice-weekly and three once-weekly regimens in the initial treatment of pulmonary tuberculosis. Bull. Wld Hlth Org. 43, 143.

Tuberculosis Chemotherapy Centre, Madras. (1973). Controlled comparison of oral twice-weekly and oral daily isoniazid plus PAS in newly-diagnosed pulmonary tuberculosis. Brit. med. J. ii, 7.

WHO Collaborating Centre for Tuberculosis Chemotherapy, Prague. (1971). A comparative study of daily and twice-weekly continuation regimens of tuberculosis chemotherapy, including a comparison of two durations of sanatorium treatment. Bull. Wld Hlth Org. 45 573. 